\title{
Optical second-harmonic generation and scanning tunneling microscopy study of the self-assembly process of cyanine dyes on $\mathrm{Br}-\mathrm{Ag}(111)$ substrates
}

\author{
I. Drevenšek-Olenik, ${ }^{1}$ F. Touhari, ${ }^{2}$ J. W. Gerritsen, ${ }^{2}$ P. Callant, ${ }^{3}$ Th. Rasing, ${ }^{2}$ and H. van Kempen ${ }^{2}$ \\ ${ }^{1}$ Department of Physics, University of Ljubljana, Jadranska 19 and J. Stefan Institute, Jamova 39, SI-1000 Ljubljana, Slovenia \\ ${ }^{2}$ Research Institute for Materials, University of Nijmegen, Toernooiveld1, 6525 ED, Nijmegen, The Netherlands \\ ${ }^{3}$ Agfa-Gevaert N. V., Septestraat 27, B-2640 Mortsel, Belgium \\ (Received 15 May 2001; revised manuscript received 19 September 2001; published 19 February 2002)
}

\begin{abstract}
Surface optical second-harmonic generation (SSHG) was used to monitor in situ the adsorption kinetics of cyanine dyes on bromide modified $\mathrm{Ag}(111)$ substrates and to probe the structural ordering and orientation of the self-assembled aggregates. The observed SSHG changes are compared to the nanoscopic configuration of the aggregates determined by scanning tunneling microscopy and to the macroscopic degree of the surface coverage that was deduced from optical absorption measurements. Due to substrate-adsorbate interaction, the nonlinear optical response of the surface covered with ordered aggregates is significantly different from the case of disordered adsorption. Our observations demonstrate that SSHG can be used to identify the character of molecular self-aggregation on model silver-halide surfaces.
\end{abstract}

DOI: 10.1103/PhysRevB.65.115404

PACS number(s): 68.08.-p, 78.68.+m, 68.37.-d

\section{INTRODUCTION}

Adsorption of cyanine dyes on the surface of silver halide microcrystals in appropriate conditions leads to formation of ordered two-dimensional molecular aggregates with a typical size of several tens of nanometers. The aggregation process is associated with the appearance of a narrow excitonic band in the optical absorption spectrum, which makes the aggregates suitable for applications in spectral sensitization of photographic films and in holographic emulsions. ${ }^{1,2}$ It has been observed recently, that a similar ordered aggregation can also occur on sputter-grown $\mathrm{Ag}$ or $\mathrm{Au} / \mathrm{Ag}$ films covered with an adsorbed halide monolayer. ${ }^{3}$ These model surfaces imitate structurally and chemically the properties of silver halide grains and in addition, on account of their conductivity and flatness, provide also a possibility to study the aggregates by means of scanning tunneling microscopy (STM) and other surface probe techniques. ${ }^{4}$

When using model surfaces, an important question is what is the correlation between the aggregation features of a specific dye on the silver halide grains and on the model surface. A more general problem is how the aggregate structure depends on the chemical composition of the dye and on the surface quality of the substrate. STM studies of model surfaces revealed the formation of the brick-stone arranged $J$ and $H$ aggregates as well as the herringbone structure, but in several cases also more complex forms were found. The nanoscopic molecular arrangement was usually observed to be in agreement with the sign of the shift of the adsorbate absorption peak. ${ }^{3}$ The widths and positions of the absorption peaks were, nevertheless, often found to be significantly different than on silver halide grains. ${ }^{4}$ The structural modifications related to these differences are not yet explained.

The difficulty of determing the aggregate absorption spectra on model surfaces is their very low signal in spectroscopic reflectance with respect to the underlying metallic film. In view of this problem the aggregation process can be more suitably probed by nonlinear reflection, i.e., surface optical second-harmonic generation (SSHG). SSHG derives its surface sensitivity from the symmetry breaking at interfaces and is a very appropriate technique for the investigation of adsorption processes. As it is described by a higher rank tensor, it is also more sensitive to surface structure and molecular orientation than the linear reflection. ${ }^{5}$ SSHG can, for example, provide information on location of the binding sites of the adsorbed molecules, ${ }^{6}$ which is difficult to be resolved from the STM and absorption studies.

During the last two decades SSHG has been used to investigate the aggregation phenomena in Langmuir-Blodgett (LB) films of various cyanine dyes probed either in situ at the air-water interface or transposed onto solid substrates. ${ }^{7-11}$ It was observed that aggregates within the LB films form numerous small domains with a noncentrosymmetric polar structure. However, the SSHG from aggregates adsorbed directly onto silver halides or other solid substrates was to our knowledge not investigated yet.

In this paper we report on a comparative study of the self-aggregation of cyanine dyes on model $\mathrm{AgBr} / \mathrm{Ag}$ surfaces by combining STM, linear absorption and SSHG measurements. A primary motivation of our work was to find a connection between the SSHG response and the microscopic structure of the aggregates and consequently exploit the applicability of SSHG for structural determination of aggregates and the aggregation process. Two slightly different thiocyanines were investigated (Fig. 1). As the resonance absorption frequencies of these molecules are well separated from the optical frequencies used in our experiment, our measurements probed predominantly the adsorption induced changes in the electronic states of the underlying Ag film. ${ }^{12}$ These changes are determined by the charge transfer process between the adsorbate and the substrate, which is of high importance in all kinds of photosensitization applications. 


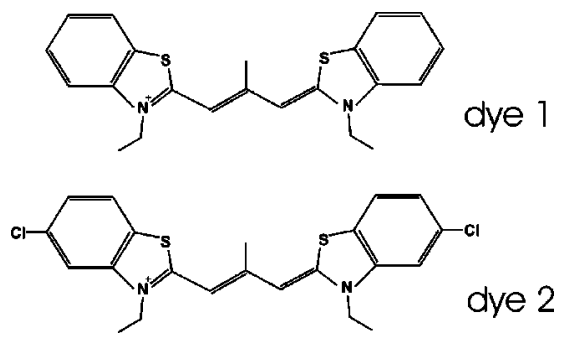

FIG. 1. Chemical structure of the cyanine dyes used in our measurements. Their monomer absorption spectra measured in 1:1 water-ethanol solution exhibit maxima at 540 and $550 \mathrm{~nm}$, respectively.

Our results show that formation of aggregates leads to a large phase shift and a significant decrease of the azimuthaly isotropic part of the SSHG response with respect to the anisotropic one. In case of disordered monomer adsorption, on the contrary, both SSHG contributions decrease very similarly. These observations can be explained by the interaction between the adsorbate and the substrate, which induces different modifications of the in-plane and the out-of-plane components of the surface nonlinear optical susceptibility.

\section{EXPERIMENTAL}

\section{A. Sample preparation}

Dye 1 (Fig. 1) forms a so-called herringbone structure on $\mathrm{AgBr}$ emulsion microcrystals, whereas dye 2, differing from dye 1 only by chloride groups on both ends, leads to the formation of the $H$ aggregates. ${ }^{13}$ We followed a procedure developed by Kawasaki and $\mathrm{Ishii}^{3}$ to prepare a $\mathrm{AgBr} / \mathrm{Ag}$ model surface. A $240 \mathrm{~nm}$ thick Ag layer was evaporated at a rate of $1 \mathrm{~nm} / \mathrm{s}$ on a freshly cleaved mica sheet heated at $200{ }^{\circ} \mathrm{C}$ under a pressure of $10^{-7}$ Torr. The evaporation was followed by an annealing for $1 \mathrm{~h}$ at $200^{\circ} \mathrm{C}$, which led to the formation of atomically flat terraces more than $100 \mathrm{~nm}$ wide as seen by STM. The resulting (111) crystallographic orientation was confirmed by $\mathrm{x}$-ray measurements.

Two ways to prepare the dye adsorbates were used: in the so-called one-step coating procedure, freshly evaporated Ag substrates were immersed for $45 \mathrm{~min}$ in a mixture of dye $\left(10^{-4} \mathrm{M}\right)$ and $\mathrm{KBr}\left(10^{-3} \mathrm{M}\right)$ in a $1: 1$ water/ethanol solution. Samples were then rinsed with cold water to remove the non-adsorbed molecules and dried under a flow of nitrogen. In the two-step coating procedure the freshly evaporated Ag films were first immersed for $45 \mathrm{~min}$ in a solution of 1:1 water/ethanol with $10^{-3} \mathrm{M} \mathrm{KBr}$. After rinsing and drying, they were probed by SSHG, which took about $30 \mathrm{~min}$ altogether. Afterwards these $\mathrm{AgBr} / \mathrm{Ag}(111)$ substrates were covered with the selected dye using a 1:1 water/ethanol solution with $10^{-4} \mathrm{M}$ of the dye. SSHG measurements were repeated on the same sample part before and after immersion in the dye solution. In addition, in situ adsorption experiments were performed, with the sample immersed in the dye solution.

\section{B. Measurements}

SSHG experiments were performed using a pulsed $\mathrm{Ti}$ : sapphire laser (100 fs, $76 \mathrm{MH}, 800 \mathrm{~nm}$ ) with an average power of $250 \mathrm{~mW}$ focused onto the sample to a spot size of about $0.3 \mathrm{~mm}$. The incident angle of the fundamental beam was $45^{\circ}$. After proper spatial and spectral filtering the outcoming specularly reflected SHG light was detected by a photomultiplier connected to a photon counter. A typical accumulation time of the $\mathrm{SH}$ light was $1 \mathrm{~s}$. The sample was mounted on a rotation stage either in air or within a flow box with dry nitrogen and was kept at room temperature. The SSHG signal was tested to be stable over the time of several tens of minutes, confirming that on the corresponding time scale there was no essential laser induced bleaching and no significant desorption of the bromide layer nor of the cyanine molecules. A possible fast desorption, on a time scale below the accumulation time of the SH light, can be rejected by the observation that very similar SHG signals were observed from freshly illuminated sample parts and from sample parts which were exposed a long time.

Absorption spectra of the aggregates on the $\mathrm{AgBr} / \mathrm{Ag}$ (111) model surface were measured using a Shimadzu Diffuse Reflectance spectrometer equipped with a halogen lamp (360-800 nm) and a deuterium lamp (240-360 nm). The optical absorbance of the deposited layer was obtained by subtracting the absorbance of the Ag film.

STM images were taken with a home made microscope especially designed for imaging molecular layers ${ }^{14}$ as it allows in particular to work with low tunneling currents, typically 10 to $30 \mathrm{pA}$. Such currents are necessary to minimize tip-surface interactions and thus minimizing possible damages of the molecular layer. The STM was operated in the constant current mode with $\mathrm{Pt} / \mathrm{Ir}$ cut wires as the tip.

\section{Data analysis procedures}

The nonlinear optical response of the $\mathrm{Ag}(111)$-air interface at fundamental frequencies below the interband transition $(3.8 \mathrm{eV})$ exhibits a strong rotational anisotropy, which is presumably related to the electronic band structure of $\mathrm{Ag}$ in the vicinity of the Fermi level. ${ }^{15}$ The azimuthal dependencies of the intensity of the specularly reflected second-harmonic beam for various polarization combinations are given by ${ }^{16,17}$

$$
\begin{gathered}
I_{p p}(2 \omega) \propto\left|\chi_{p p p}^{(2)}\right|^{2} I^{2}(\omega)=\mid A_{p p}+B_{p p} e^{\left.i \delta_{p p} \cos (3 \phi)\right|^{2},} \\
I_{p s}(2 \omega) \propto\left|\chi_{p s s}^{(2)}\right|^{2} I^{2}(\omega)=\left|B_{p s} \sin (3 \phi)\right|^{2}, \\
I_{s p}(2 \omega) \propto\left|\chi_{s p p}^{(2)}\right|^{2} I^{2}(\omega)=\mid A_{s p}+B_{s p} e^{\left.i \delta_{s p} \cos (3 \phi)\right|^{2},} \\
I_{s s}(2 \omega) \propto\left|\chi_{s s s}^{(2)}\right|^{2} I^{2}(\omega)=\left|B_{s s} \sin (3 \phi)\right|^{2},
\end{gathered}
$$

where $\chi_{i j k}^{(2)}$ represent the corresponding effective non-linear optical susceptibilities, $I(\omega)$ the intensity of the incident fundamental optical beam, and $\phi$ the angle between the [2 $\overline{11}]$ crystal axis and the plane of incidence. The subscripts refer to polarizations of the fundamental and the second harmonic beams and denote directions parallel ( $p$ polarization) and perpendicular ( $s$ polarization) to the plane of incidence re- 
spectively. The relations between $A_{i j}, B_{i j}, \delta_{i j}$ and the various nonlinear polarization sources of the Ag crystal are described in the paper of Sipe, Moss, and van Driel. ${ }^{16}$

Adsorption of the dye molecules onto the $\operatorname{Ag}(111)$ surface induces a perturbation of its nonlinear optical susceptibility which converts to ${ }^{18}$

$$
\tilde{\chi}_{i j k}^{(2)}=\chi_{i j k}^{(2)}+\chi_{i j k, \text { int }}^{(2)}+\chi_{i j k, \text { ads }}^{(2)},
$$

where $\chi_{i j k}^{(2)}$ represents the intrinsic contribution of the substrate, $\chi_{i j k, \text { int }}^{(2)}$ a contribution induced by the substrateadsorbate interaction, and $\chi_{i j k \text {,ads }}^{(2)}$ the intrinsic contribution of the dye molecules. As we found that the SSHG signal from a thin film of the dye molecules adsorbed on a fused silica plate using the same excitation wavelength of $800 \mathrm{~nm}$ was negligibly small, the last term is expected to be not important in our experiments. The observed changes in the SSHG response, resulting from modifications of the parameters $A_{i j}$, $B_{i j}$, and $\delta_{i j}$ in Eqs. (1)-(4), are therefore related to the interaction between the adsorbate and the substrate. The most systematic and reproducible results were obtained for $p-p$ and $s-s$ polarization combinations, therefore we limited our analysis to these two combinations. Measurements of the SSHG response as a function of the azimuthal angle $\phi$ were performed in the full range of $0^{\circ}<\phi<360^{\circ}$ and a threefold rotational symmetry was always observed. The fitting of the data was also performed on the full azimuthal range, while for clarity reasons the figures give only the results for $0^{\circ}$ $<\phi<120^{\circ}$.

\section{RESULTS}

\section{A. SSHG measurements}

Figure 2 shows the dependencies of the SSHG signal on the azimuthal angle $\phi$ for a freshly prepared $\operatorname{Ag}(111)$ substrate and for a bromide converted $\operatorname{Ag}(111)$ substrate. The presence of the $\mathrm{AgBr}$ monolayer induces only relatively minor variations of the SSHG signal compared to the bare $\mathrm{Ag}$ film: the $I_{p p}(2 \omega)$ on average slightly increases, while the $I_{s s}(2 \omega)$ slightly decreases. The corresponding modifications of the fit parameters $A_{p p}, B_{p p}, B_{s s}$, and $\delta_{p p}$ are listed in Table I.

Figure 3 shows a variation of $I_{p p}(2 \omega)$ at $\phi=0^{\circ}$ measured in situ during adsorption of dye 1 on the $\operatorname{Ag}(111)$ substrate. The aggregation features observed in this experiment were similar as in the so-called one-step coating procedure. The sample was mounted in the center of a cylindrical container filled with the $\mathrm{KBr} /$ water/ethanol mixture and the SSHG response was monitored for about $10 \mathrm{~min}$. After that, at $t=0$, a few drops of concentrated solution of dye 1 were added to the container and the resulting mixture was carefully stirred. A subsequent decrease of $I_{p p}(2 \omega)$ was observed on a time scale of about $30 \mathrm{~min}$. The absorption spectrum of dye 1 in water/ethanol solution has a peak at $542 \mathrm{~nm}$, while the absorbances at 400 and $800 \mathrm{~nm}$ are negligible, ${ }^{13}$ hence the observed decrease of $I_{p p}(2 \omega)$ can be related predominantly to the modification of the SSHG response of the Ag film.

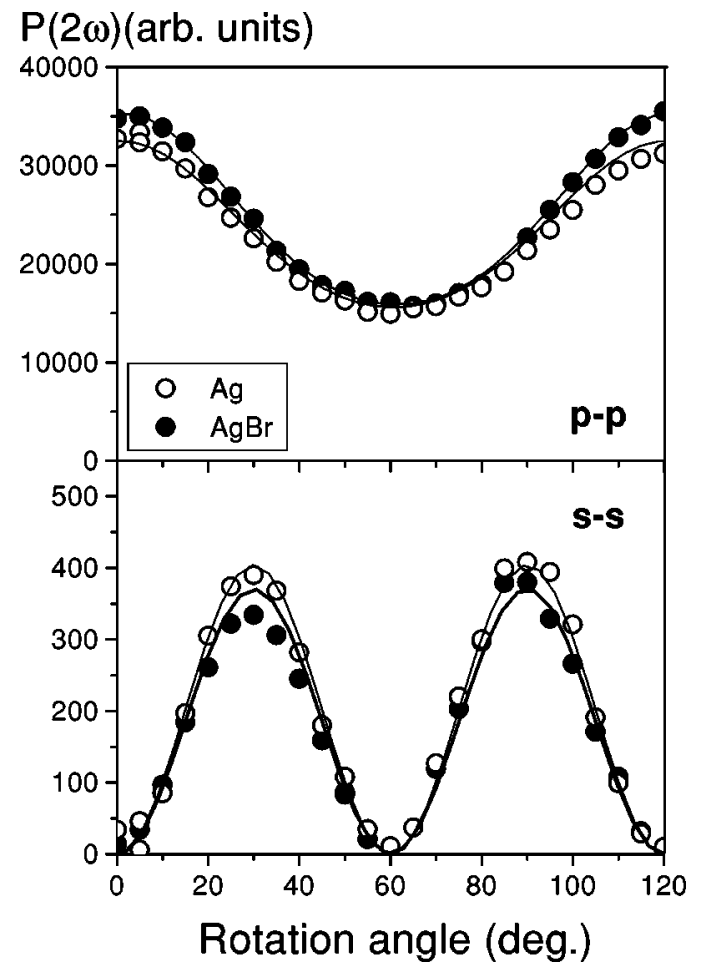

FIG. 2. Second harmonic intensity $I(2 \omega)$ for $p-p$ and $s-s$ polarization combinations as a function of the azimuthal angle $\phi$ for a freshly prepared $\operatorname{Ag}(111)$ substrate (open symbols) and for a bromide converted $\operatorname{Ag}(111)$ substrate (solid symbols). Solid lines are fits to Eqs. (1) and (4), respectively.

Azimuthal measurements of the $I(2 \omega)$ for the one-step coating procedure (Fig. 4) show that both the $p-p$ and the $s-s$ response significantly reduce. The isotropic term $A_{p p}$ and the anisotropic terms $B_{p p}$ and $B_{s s}$ appear to be affected by the adsorption process in a very similar manner, i.e., they all decrease for about $20 \%$. The parameter $\delta_{p p}$ is shifted about $10^{\circ}$ (see Table I).

An essentially different modification of the $I(2 \omega)$ is obtained, if the sample is prepared by the two-step coating procedure. As shown in Fig. 5, in this case only the $p-p$ SSHG signal considerably reduces, while the $s-s$ signal remains practically unchanged. Consequently only the isotropic term $A_{p p}$ decreases for about $20 \%$, while $B_{p p}$ and $B_{s s}$ retain their values. The parameter $\delta_{p p}$ exhibits a significant shift from $\delta_{p p}=39^{\circ}$ to $\delta_{p p}=0^{\circ}$ (see Table I).

The fit parameters for different samples are collected in Table I. The data are given relatively with respect to the value of $A_{p p}$ for a fresh $\mathrm{Ag}(111)$ substrate. The results obtained for dye 2 are very similar to those for dye 1 .

\section{B. Absorption spectroscopy}

Due to the similarities between the absorption properties of the two dyes, also in this section only the results for dye 1 are presented. Absorption spectra of samples prepared by the two-step coating procedure indicate the presence of ordered aggregates (Fig. 6). Indeed, we found a peak at $516 \mathrm{~nm}$ as well as a shoulder around $570 \mathrm{~nm}$, values close to those obtained for $\mathrm{AgBr}$ octahedral microcrystals in an emulsion 
TABLE I. Values of fit parameters [Eqs. (1), (4)] for various samples.

\begin{tabular}{lccccc}
\hline \hline Sample & Procedure & $A_{p p}$ & $B_{p p}$ & $\delta_{p p}$ & $B_{s s}$ \\
\hline $\mathrm{Ag}(111)$ & & $1.00 \pm 0.01$ & $0.24 \pm 0.04$ & $39^{\circ} \pm 9^{\circ}$ & $0.13 \pm 0.01$ \\
$\mathrm{AgBr} / \mathrm{Ag}(111)$ & & $1.03 \pm 0.03$ & $0.27 \pm 0.02$ & $39^{\circ} \pm 5^{\circ}$ & $0.13 \pm 0.01$ \\
dye1/AgBr/Ag(111) & one-step coating & $0.82 \pm 0.02$ & $0.17 \pm 0.01$ & $23^{\circ} \pm 5^{\circ}$ & $0.09 \pm 0.01$ \\
dye2/AgBr/Ag(111) & one-step coating & $0.81 \pm 0.03$ & $0.19 \pm 0.01$ & $33^{\circ} \pm 4^{\circ}$ & $0.10 \pm 0.01$ \\
dye1/AgBr/Ag(111) & two-step coating & $0.84 \pm 0.02$ & $0.23 \pm 0.03$ & $0^{\circ} \pm 13^{\circ}$ & $0.13 \pm 0.01$ \\
dye2/AgBr/Ag(111) & two-step coating & $0.82 \pm 0.02$ & $0.21 \pm 0.04$ & $0^{\circ} \pm 17^{\circ}$ & $0.14 \pm 0.01$ \\
\hline \hline
\end{tabular}

(532 and $570 \mathrm{~nm}$ ). The presence of two absorption bands is the signature of a herringbone structure. ${ }^{13}$

For samples prepared by the one-step coating procedure the amount of monomer molecules on the surface was much larger. Correspondingly we observed practically entirely a monomerlike peak close to $540 \mathrm{~nm}$ (Fig. 6). This means that there were no, or only very little, ordered aggregates present on the surface, as the shoulder around $570 \mathrm{~nm}$ is very weak. $^{13}$

\section{STM measurements}

As the molecules are less than $1 \mathrm{~nm}$ height, a high resolution STM picture is difficult to get when scanning a large area. Therefore, rather small areas were imaged on many different places at the sample surface to get a qualitative insight in the degree of ordering. Typical examples of observations of the largest areas where the molecular aggregates can still be observed are given in Figs. 7(a) and 7(b). Figure 7(a) corresponds to a sample prepared by the two-step coating procedure. Large and smooth terraces can be seen which are covered by a monolayer of dyes forming aggregates (see arrow). These consist in rows of molecules which follow the (111) orientation of the surface. This kind of image is typical for the whole surface.

On the other side, an equivalent area imaged for a sample prepared by the one-step coating procedure [Fig. 7(b)] shows a bad molecular coverage, as only a few aggregates domains

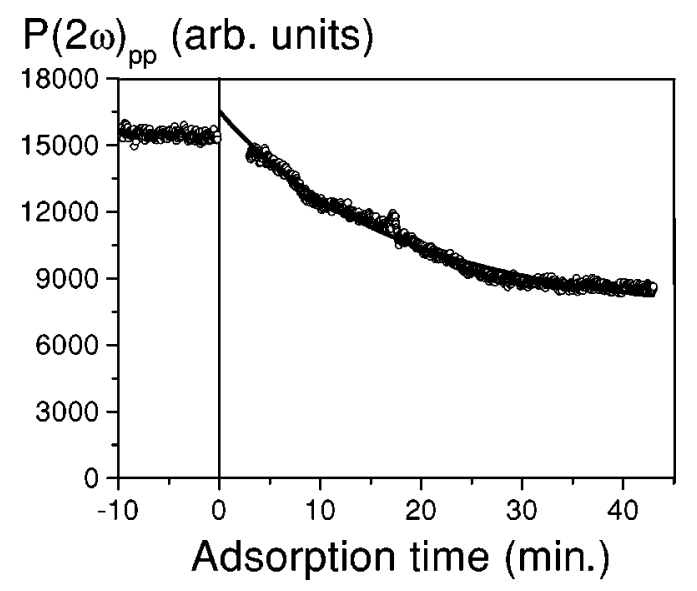

FIG. 3. Variation of the $p-p$ SSHG signal at $\phi=0$ measured during adsorption of the dye 1 onto $\mathrm{AgBr} / \mathrm{Ag}(111)$ substrate. The dye was added to the solution at $t=0$. Solid line is a fit to Eq. (6). can be seen. For this kind of sample we often found large areas that did not show any indication of ordered aggregation at all. The observed surface structures are hence in agreement with the absorption spectra.

\section{DISCUSSION}

Exposure of $\mathrm{Ag}(111)$ films to ambient conditions leads to an immediate coverage of the film with a native hydroxide monolayer. By immersion in a $\mathrm{KBr}$ solution this monolayer is substituted by $\mathrm{Br}$ anions which form a quasi- $\sqrt{3}$ $\times \sqrt{3} / R 30^{\circ}$ structure with respect to the underlying $\mathrm{Ag}$ (111) lattice. ${ }^{3}$ In this substitution process the surface structure and conducting electron density of the film remain the same and consequently the nonlinear optical response is expected to

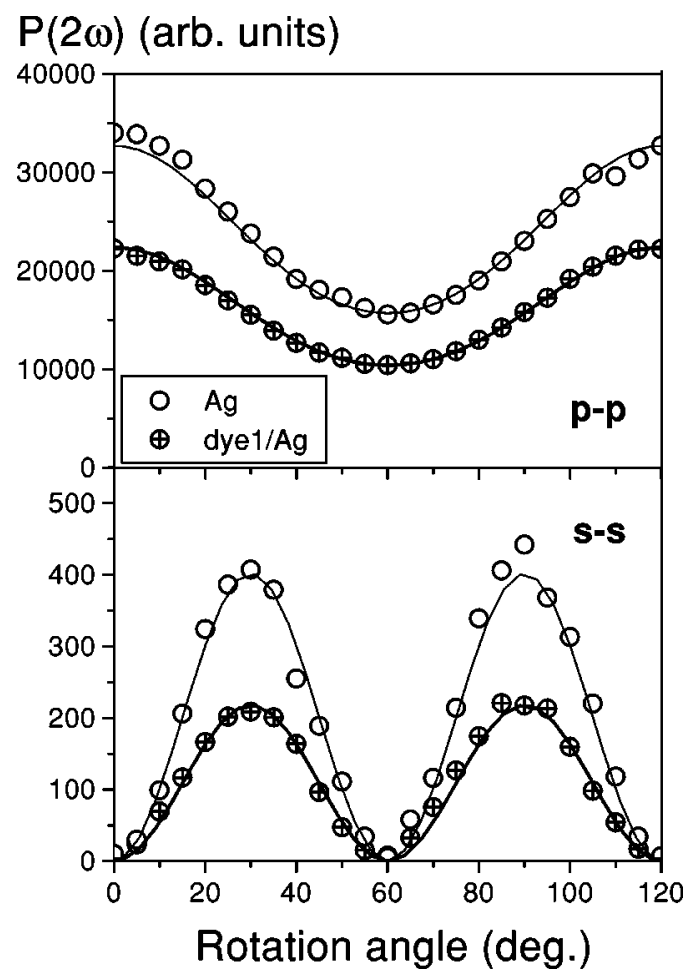

FIG. 4. Second harmonic intensity $I(2 \omega)$ for $p-p$ and $s-s$ polarization combinations as a function of the azimuthal angle $\phi$ measured for a freshly evaporated $\operatorname{Ag}(111)$ substrate (open symbols) and for $\operatorname{Ag}(111)$ substrate covered with dye 1 by one-step immersion procedure (symbols with crosses). Solid lines are fits to Eqs. (1) and (4), respectively. 


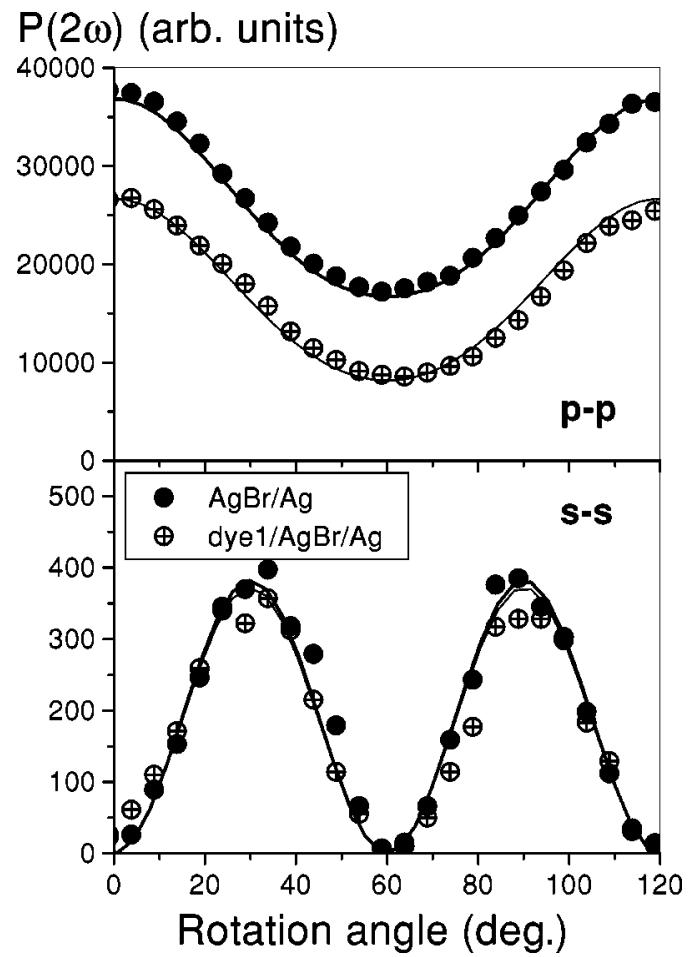

FIG. 5. Second harmonic intensity $I(2 \omega)$ for $p-p$ and $s-s$ polarization combinations as a function of the azimuthal angle $\phi$ for $\mathrm{AgBr} / \mathrm{Ag}(111)$ substrate (open symbols) and for the same substrate covered with dye 1 by the two-step coating procedure (symbols with cross). Solid lines are fits to Eqs. (1) and (4), respectively.

remain unaffected, as is consistent with our observations (Fig. 2). Due to charge transfer between the adsorbate and the substrate, the nonlinear optical properties of the substituted $\mathrm{Br}-\mathrm{Ag}(111)$ surface are, however, significantly different from the case of a chemically clean UHV prepared $\mathrm{Ag}(111)$ single crystal. ${ }^{19}$ The value of $B_{p p} / A_{p p}$ (Table I) is about five times lower than observed at similar wavelengths on a clean $\operatorname{Ag}(111)$ surface and the relative phase $\delta_{p p}$ is also different.

The adsorbed $\mathrm{Br}^{-}$anions are supposed to stimulate the aggregation of the cyanine molecules on the $\operatorname{Ag}(111)$ surface.

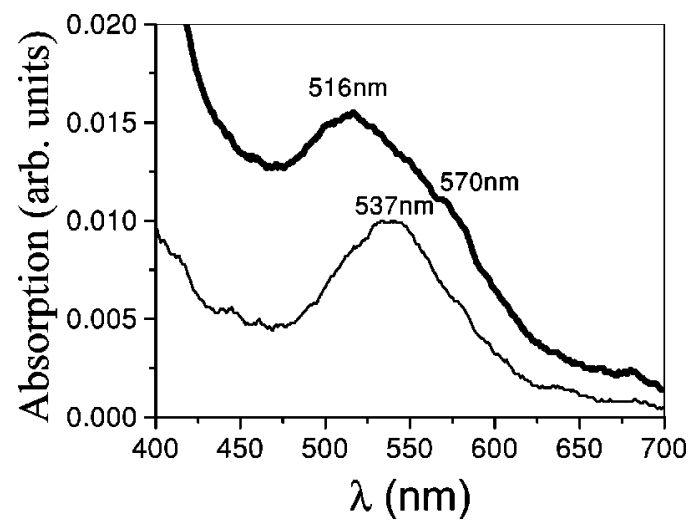

FIG. 6. Absorption spectra of dye 1 adsorbed on a $\mathrm{AgBr} / \mathrm{Ag}$ substrate by a two-step coating procedure (thick line) and by the one-step coating procedure (thin line). Strong absorption for wavelengths below $450 \mathrm{~nm}$ is the effect of the $\mathrm{AgBr}$ layer.

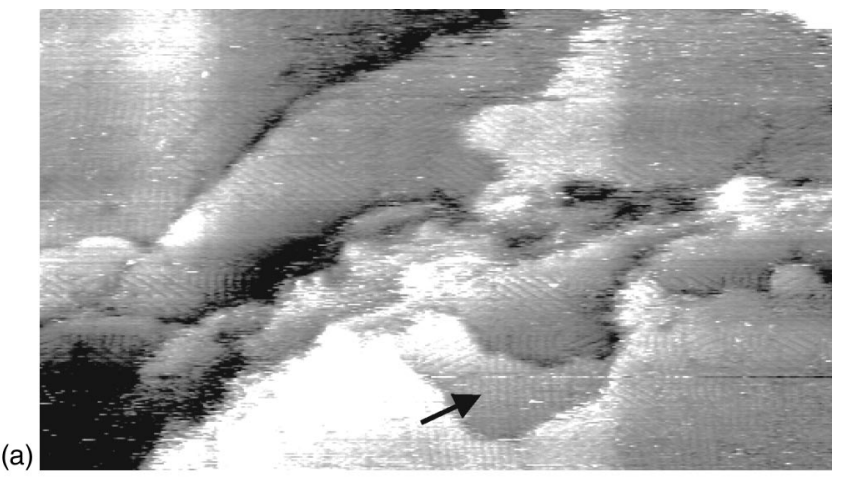

(b)

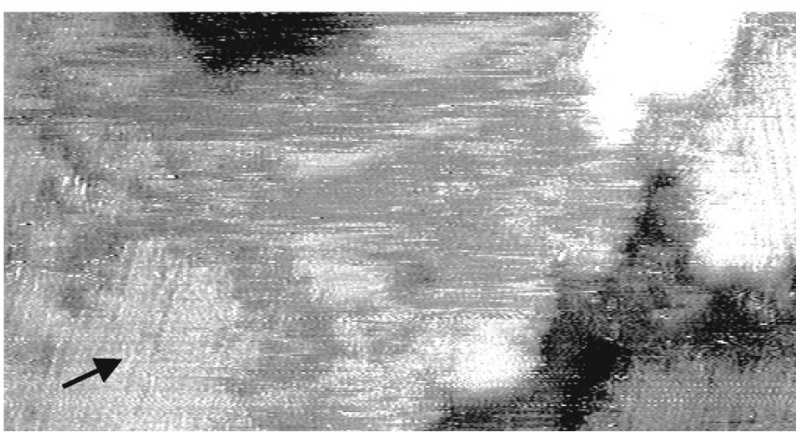

FIG. 7. STM images $\left(100 \times 55 \mathrm{~nm}^{2}\right.$ region $)$ of the aggregates of dye 1 prepared by two-step coating procedure (a) and by one-step coating procedure (b).

That is because $\mathrm{Br}^{-}$occupies on the $\mathrm{Ag}(111)$ surface presumably the same sites as on the surface of the AgBr microcrystals, on which cyanine dye molecules predominantly aggregate. As evident from Fig. 3, an essential reduction of the SSHG appears when the dye is added to the solution, which can be associated with the modification of the surface nonlinear optical response due to the interaction of the dye molecules with the substrate. Changes related to modification of the refractive index of the solution can be neglected, as also follows from a comparison with the data for a similar sample held in air (see Fig. 4).

The corresponding adsorption kinetics is expected to exhibit a Langmuir type behavior that can be written as ${ }^{20}$

$$
I_{p p}(2 \omega, t) \propto\left|1-\left(\chi_{p p p, \text { int }}^{(2)} / \chi_{p p p}^{(2)}\right)\left(1-e^{-t / \tau}\right)\right|^{2} I^{2}(\omega) .
$$

The fit of the experimental data to Eq. (6) is presented as a solid line in Fig. 3. The agreement is quite good and gives a characteristic adsorption time $\tau=22 \pm 2 \mathrm{~min}$. This value is in agreement with the results of Kawazaki et al. ${ }^{3}$ who found that the absorption intensity of aggregates on a $\mathrm{AgBr} / \mathrm{Ag}$ model surface is maximized after 20 min immersion and afterwards saturates. The ratio of the interaction susceptibility to the intrinsic susceptibility is real and has a value of $\left(\chi_{p p p, \text { int }}^{(2)} / \chi_{p p p}^{(2)}\right)=0.33 \pm 0.01$. The corresponding zero phase shift between the $\chi_{p p p \text {,int }}^{(2)}$ and $\chi_{p p p}^{(2)}$ is in agreement with the results of the azimuthal SSHG measurements, which also showed only relatively small changes of the parameter $\delta_{p p}$ for the one-step coating procedure (Fig. 4, Table I).

In a disordered adsorption process the dye molecules occupy random positions and azimuthal orientations with re- 
spect to the underlying crystal lattice of the substrate. The interaction potential between the adsorbate and the substrate consequently exhibits the same $C_{3 v}$ symmetry as the surface of the substrate. The azimuthal dependencies of the modified SSHG signal [Eq. (5)] thus retain the forms as given in Eqs. (1)-(4).

A different situation could appear in the two-step coating procedure when ordered aggregates are formed with an internal structure of lower symmetry than the substrate. If aggregate domains are arranged equivalently with respect to the six analogous crystallographic orientations of the topmost layer of the $\operatorname{Ag}(111)$, the modification of azimuthal dependencies of $I(2 \omega)$ is given as

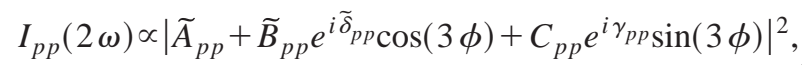

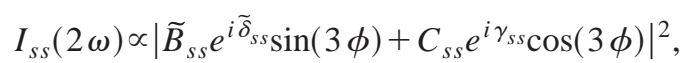

where $C_{p p}, C_{s s}$ and $\gamma_{p p}, \gamma_{s s}$ represent additional terms with respect to the uncoated substrate [Eqs. (1)-(4)]. In our experiments, however, the values of $C_{p p}$ and $C_{s s}$ were always found to be zero, which indicates that the interaction potential between the substrate and the adsorbate exhibits a mirror plane symmetry that is common to both the azimuthal angular distribution of the adsorbed molecules and the underlying crystal lattice. This is a vertical mirror plane along the [2 $\overline{11}]$ crystal axis of the Ag. In herringbone aggregates associated with the dye 1 the rows of the molecules observed in the STM images [Fig. 7(a)] therefore coincide either with the [2ㅍ] directions of the $\mathrm{Ag}$ or with their intermediates.

The SH response from a metal is generated by three different sources: a bulk current, a surface current perpendicular to the boundary, and a surface current parallel to the boundary. ${ }^{21}$ The bulk current originates from the skin depth region typically several tens of nanometers thick and is consequently not very sensitive to the details of the surface structure. The surface currents are generated within a tenth to a few nanometers thick outmost layer of the metal and are hence much more strongly affected by modifications of the surface electronic density induced, for example, by the presence of various adsorbates. ${ }^{15,22}$ The surface sensitivities of perpendicular and parallel surface currents, nevertheless, display quite different aspects. The perpendicular surface current, which is related to the azimuthally isotropic part of the SSHG, depends primarily on the shape of the surface potential barrier in the normal direction, while it is relatively insensitive to the arrangement of the adsorbed molecules in the tangential direction. ${ }^{23}$ The parallel surface current, related to anisotropic SSHG, is on the other hand strongly affected by the tangential arrangement of the adsorbates. ${ }^{1524}$ Adsorbates with irregular or incommensurate structure with respect to the underlying metal film cause an increased surface electron scattering which results in a strong decrease of the tangential SH response. ${ }^{25}$

For both types of samples studied in our experiments we observed a very similar reduction (for about $20 \%$ ) of the isotropic SSHG parameter $A_{p p}$ due to the adsorption of the dye (Table I). Such a result suggests that the adsorption in- duced modification of the surface potential barrier in the normal direction is not very sensitive to the way of the growth of the dye $/ \mathrm{AgBr}$ adlayer. The dye molecules and their counterions seem to equivalently compete with $\mathrm{Br}^{-}$and $\mathrm{K}^{+}$ions in screening of the surface sites of the $\operatorname{Ag}(111)$ film. This competition can be a reason for the formation of very disordered aggregates in the case of a one-step coating procedure, as a mixed instead of a separated double surface adlayer is formed.

The anisotropic SSHG parameters $B_{p p}$ and $B_{s s}$ show a pronounced difference between the two sample preparation procedures. In the one-step coating procedure the values of $B_{p p}$ and $B_{s s}$ are significantly reduced (Table $\mathrm{I}$ ), which is in accordance with the disordered dye aggregation detected by STM. In the two-step coating procedure, on the contrary, the variations of $B_{p p}$ and $B_{s s}$ are much smaller, in agreement with the observation that this procedure is associated to the formation of large ordered aggregates.

A significant difference between the two sample preparation procedures was observed also in the parameter $\delta_{p p}$. In the one-step coating the shift of $\delta_{p p}$ is much smaller than in the two-step coating (Table I). This can be related to the difference in the nature of the adsorption process, i.e., the difference of the effects on the nonlinear susceptibility due to physisorption versus chemisorption. ${ }^{20}$ The large shift of $\delta_{p p}$ observed in two-step coating procedure suggests that ordered aggregation of cyanine dyes on $\mathrm{Br}-\mathrm{Ag}(111)$ occurs predominantly via chemisorption, which very probably takes place via bonding of the surface silver-bromide layer with the sulfur atoms, similar to the organosulfur monolayers formed on gold $^{6}$.

\section{CONCLUSIONS}

By monitoring the SSHG changes occurring during adsorption of the thiocyanine dyes on a model $\mathrm{AgBr} / \mathrm{Ag}(111)$ surface we directly determined the optimal immersion time for formation of a complete monolayer. Our result is in good agreement with the data reported in the literature. We found that two slightly different procedures of preparation of the adlayers gave very different results in terms of coverage of the surface by ordered aggregates. For two dyes studied, the STM images showed large ordered aggregates and very little monomer on the surface if precursory modification of the substrates with $\mathrm{KBr}$ was used. On the other hand, when the sample was made in a one-step coating procedure by immersion into a mixed dye/ $\mathrm{KBr}$ solution, we observed a large fraction of monomers and only very small ordered aggregates. The difference between the two kinds of aggregation processes clearly showed up in the accompanied modification of the SSHG response, which can hence be used to probe the adsorbate ordering features on a macroscopic scale.

The STM images showed that, similar to the LB films of cyanines on water, the aggregates adsorbed on a $\mathrm{AgBr} /$ $\mathrm{Ag}$ (111) surface form domains with a stripelike internal arrangement. In addition, the SSHG measurements revealed that the orientational angular distribution of the dye molecules within the domains exhibits a mirror plane symmetry 
along the $[2 \overline{11}]$ crystal axis of the underlying $\operatorname{Ag}(111)$ lattice. Further experiments, involving resonant excitation of the dye molecules and phase measurements, could address the polarity of the molecular orientation, i.e., the question which side of the cyanine molecule is directly bounded to the substrate and which side is pointing upwards from the surface.

\section{ACKNOWLEDGMENTS}

This work was partly supported by the EU networks EMIT (Contract No. ERB-FMRX-CT98-0198) and SILC (Contract No. ERB FMRXCT 980209). We are grateful to J. Louwet from Agfa-Gevaert N.V. (Mortsel, Belgium) for performing the absorption measurements.
${ }^{1}$ G.R. Bird, K.S. Norland, A.B. Rosenoff, and H.B. Michaud, Photograph. Sci. Eng. 12, 196 (1968).

${ }^{2}$ A. Hertz, in Theory of the Photographic Process, 4th ed., edited by T.H. James (Macmillan, New York, 1977), pp. 235-250.

${ }^{3}$ M. Kawasaki and H. Ishii, Langmuir 11, 832-841 (1995); J. Imaging Sci. Technol. 39, 210 (1995); M. Kawasaki, H. Ishii, and H. Uchiki, Microsc. Res. Tech. 42, 100 (1998).

${ }^{4}$ G. Janssens, F. Touhari, J. W. Gerritsen, H. van Kempen, P. Callant, G. Deroover, and D. Vandenbroucke, Chem. Phys. Lett. 344, 1 (2001).

${ }^{5}$ T.F. Heinz, in Nonlinear Surface Electromagnetic Phenomena, edited by H.E. Ponath and G.I. Stegeman (North-Holland, Amsterdam, 1991), p. 353.

${ }^{6}$ M.S. Yeganeh, S.M. Dougal, R.S. Polizzotti, and P. Rabinowitz, Phys. Rev. Lett. 74, 1811 (1995).

${ }^{7}$ F. Eisert, O. Dannenberger, and M. Buck, Phys. Rev. B 58, 10860 (1998).

${ }^{8}$ V. Mizrahi, G.I. Stegeman, and W. Knoll, Phys. Rev. A 39, 3555 (1989).

${ }^{9}$ K. Kajikawa, H. Takezoe, and A. Fukuda, Jpn. J. Appl. Phys. 30, L1525 (1991); K. Kajikawa, H. Takezoe, and A. Fukuda, Chem. Phys. Lett. 205, 225 (1993).

${ }^{10}$ S. Kobayashi, Mol. Cryst. Liq. Cryst. 217, 77 (1992).
${ }^{11}$ N. Kato, K. Saito, and Y. Uesu, Thin Solid Films 338, 5 (1999); Ferroelectrics 238, 245 (2000).

${ }^{12}$ B. Park, M.S. Kim, Y.D. Kim, E.C. Jung, and C.S. Jung, J. Phys. Chem. 97, 5080 (1993).

${ }^{13}$ Agfa-Gevaert N.V. (unpublished).

${ }^{14}$ J.W. Gerritsen, E.J.G. Boon, G. Janssens, and H. van Kempen, Appl. Phys. A: Mater. Sci. Process. 66, 79 (1998).

${ }^{15}$ A.V. Petukhov, Surf. Sci. 347, 143 (1995).

${ }^{16}$ J.E. Sipe, D.J. Moss, and H.M. van Driel, Phys. Rev. B 35, 1129 (1987).

${ }^{17}$ Y. Tang, L.J. Simpson, and T.E. Furtak, Phys. Rev. Lett. 67, 2814 (1991).

${ }^{18}$ R.M. Corn and D.A. Higgins, Chem. Rev. 94, 107 (1994).

${ }^{19}$ R.A. Bradley, R. Georgiadis, S.D. Kevan, and G.L. Richmond, J. Chem. Phys. 99, 5535 (1993).

${ }^{20}$ L. Le, L. Yanghua, Y. Gongda, W. Wencheng, and Z. Zhiming, Phys. Rev. B 40, 10100 (1989).

${ }^{21}$ J. Rudnick and E.A. Stern, Phys. Rev. B 4, 4274 (1971).

${ }^{22}$ A. Liebsch and W.L. Schaich, Phys. Rev. B 40, 5401 (1989).

${ }^{23}$ M. Weber and A. Liebsch, Phys. Rev. B 35, 7411 (1987).

${ }^{24}$ A.V. Petukhov and A. Liebsch, Surf. Sci. 334, 195 (1995).

${ }^{25}$ D.A. Koos, V.L. Shanon, and G.L. Richmond, J. Phys. Chem. 94, 2091 (1990). 